\title{
Long-term efficacy of adalimumab in the treatment of uveitis associated with juvenile idiopathic arthritis
}

This article was published in the following Dove Press journal:

Clinical Ophthalmology

30 September 2011

Number of times this article has been viewed

\author{
Kaisu Kotaniemi ${ }^{1,2}$ \\ Hanna Säilä ${ }^{2}$ \\ Hannu Kautiainen ${ }^{3}$ \\ 'Helsinki University Hospital, Helsinki, \\ Finland; ${ }^{2}$ Orton Orthopaedic Hospital \\ and Rehabilitation Unit, Helsinki, \\ Finland; ${ }^{3}$ Unit of Primary Health Care, \\ Kuopio University Hospital, Kuopio, \\ Finland
}

Background: The purpose of this study was to investigate the long-term effects of adalimumab, a tumor necrosis factor alpha antagonist, in the treatment of uveitis associated with juvenile idiopathic arthritis.

Methods: Adalimumab was initiated in 94 patients with juvenile idiopathic arthritis to treat active arthritis and/or active associated uveitis. In 18 patients, therapy was discontinued after a short period because of inefficacy or side effects. The activity of uveitis (using Standardized Uveitis Nomenclature [SUN] criteria and clinical examination) and arthritis (number of swollen or active joints) was evaluated at the start and at end of the study.

Results: At the end of the study, uveitis was under good clinical control in two thirds of 54 patients ( $31 \%$ did not need any local treatment and 35\% used only $1-2$ corticosteroid drops a day), and one third had active uveitis (at least three corticosteroid drops a day). According to SUN criteria, adalimumab treatment for uveitis showed improved activity (a two-fold decrease in uveitis activity) in $28 \%$ of patients, with a moderate response in 16 patients, no change in a further 16 patients, and worsening activity (a two-fold increase in uveitis activity) in 13\% of patients. The overall proportion of patients with active arthritis decreased. At the beginning of the study, $69 \%$ of patients with uveitis had more than two active joints, and at the end of the study only $27 \%$ had active joint disease. In 27 patients with juvenile idiopathic arthritis without uveitis on adalimumab, the number of active joints decreased from $93 \%$ to $59 \%$. Systemic corticosteroid treatment could be stopped in $22 \%$ of patients with uveitis and in $11 \%$ of those without uveitis. Most of the patients had received methotrexate, other immunosuppressive therapy, or other biological drugs before initiating adalimumab.

Conclusion: Adalimumab is a valuable option in the treatment of uveitis associated with active juvenile idiopathic arthritis.

Keywords: adalimumab, juvenile idiopathic arthritis, uveitis

\section{Introduction}

Anterior uveitis is a serious manifestation of juvenile idiopathic arthritis, with a potential for significant ocular damage and even blindness. Uveitis has been reported in $10 \%-20 \%$ of patients with juvenile idiopathic arthritis, although lower and higher figures have been published. ${ }^{1,2}$ Antinuclear antibody (ANA)-positive girls younger than seven years of age with oligoarthritis are at the greatest risk of developing uveitis. ${ }^{2}$

The first-line standard therapy for uveitis is topical and systemic corticosteroids, often reinforced by methotrexate as a second-line disease-modifying antirheumatic drug (DMARD). Tumor necrosis factor-alpha (TNF- $\alpha$ ) has been shown to play an important role in the development of uveitis. ${ }^{3}$ TNF- $\alpha$ blockers have been used to treat uveitis and 
juvenile idiopathic arthritis, and especially uveitis associated with juvenile idiopathic arthritis in children who have failed conventional topical and second-line DMARD therapy., ${ }^{4,5}$ A meta-analysis of the impact of anti-TNF therapy on anterior uveitis in ankylosing spondylitis was used to compare the effect of infliximab and etanercept to decrease the number of uveitis flares in 717 patients with ankylosing spondylitis. The flares were less frequent during infliximab than during etanercept treatment. ${ }^{6}$ A Canadian study compared the efficacy of etanercept and infliximab in uveitis among 21 patients with active uveitis at onset of treatment. A better clinical response with fewer ocular complications was seen with infliximab than etanercept. ${ }^{7}$

In a small study from Columbia, ocular inflammation in 13 of 14 children responded to adalimumab (a humanized monoclonal TNF- $\alpha$ antibody). ${ }^{8}$ A better effect of adalimumab for uveitis compared with etanercept was shown in a German study of 18 children and young adults with juvenile uveitis and/or arthritis. ${ }^{9,10}$ In a Finnish retrospective study, of 20 patients with juvenile idiopathic arthritis and chronic uveitis on adalimumab treatment, seven (35\%) showed improved activity, one (5\%) had worsening activity, and $12(60 \%)$ showed no change. ${ }^{11}$ A recent Greek study reported a decrease in uveitis flares with adalimumab treatment in three of six patients with juvenile idiopathic arthritis and uveitis. ${ }^{12}$ We describe a large retrospective cohort of patients with juvenile idiopathic arthritis and associated uveitis treated with adalimumab for a mean of two years.

\section{Patients and methods}

This study was based on data for 320 patients drawn from the juvenile idiopathic arthritis registry for biologic drugs at the Rheumatism Foundation Hospital, Heinola, Finland, for 2008-2010. Some of these cases belonged to the cohort described earlier by Tynjälä et al. ${ }^{11}$ From the patient records, we collected baseline demographic data (age, gender, age at disease onset, duration of disease), disease information (juvenile idiopathic arthritis subtype, number of active joints, laboratory values, if available), and treatment data (biologics and concomitant DMARDs, including start and stop dates, as well as reasons for withdrawal). The study was retrospective and evaluated the long-term impact of adalimumab, the monoclonal anti-TNF- $\alpha$ antibody, on juvenile idiopathic arthritis and associated uveitis.

The 2001 revised International League Against Rheumatism classification criteria for juvenile idiopathic arthritis were used in this study ${ }^{13}$ All diagnoses of juvenile idiopathic arthritis were confirmed by a pediatric rheumatologist who examined arthritis activity by evaluating the number of swollen or active joints and joints with a limited range of motion at the start of adalimumab therapy and at the end of the study. Inflammatory laboratory markers (erythocyte sedimentation rate and C-reactive protein), if available, were recorded.

Evaluation of the efficacy of adalimumab on uveitis activity was done using the Standardized Uveitis Nomenclature (SUN) criteria (a two-fold improvement or a two-fold worsening of uveitis, ie, a change in the amount of anterior chamber cells and flare) and clinical examination (evaluating the decrease in activity of inflammation in the eye by biomicroscopy, decrease in amount of anterior chamber cells and flare, and decrease in amount of topical treatment). ${ }^{14}$

Remission of juvenile idiopathic arthritis with medication was stated if the patient had no joints with active arthritis and no systemic signs (eg, fever, rash). Erythocyte sedimentation rate and $\mathrm{C}$-reactive protein needed to be at normal levels. No global assessment of disease activity scores was available in this retrospective series. ${ }^{15}$

The clinical response to treatment for uveitis was considered good if there were $<3$ anterior chamber cells/1 mm slit and there was no need for corticosteroid drops. A moderate response was deemed to be 3-9 anterior chamber cells/1 $\mathrm{mm}$ slit and no more than three corticosteroid drops per day. Ineffective treatment was considered to be $>10$ cells in the anterior chamber and more than three corticosteroid drops per day (beyond which the risk of cataract is increased).

Adalimumab was initiated in 94 patients with juvenile idiopathic arthritis because of active arthritis and/or active associated uveitis. The patients were divided into two groups, ie, 54 patients with juvenile idiopathic arthritis and uveitis and 40 patients with juvenile idiopathic arthritis and without uveitis.

The 54 patients with uveitis were either not responsive to standard immunosuppressive therapy for uveitis or were intolerant to it. Adalimumab was administered at a dose of $24 \mathrm{mg} / \mathrm{m}^{2}$ at 14-day intervals subcutaneously, and according to clinical response, up to a frequency of $24 \mathrm{mg} / \mathrm{m}^{2}$ at seven-day intervals. In 22 patients with uveitis, adalimumab was the first anti-TNF treatment and was started because of active ocular inflammation.

The results are expressed as the median \pm standard deviation and interquartile range. Statistically significant differences between the groups were evaluated using the Mann-Whitney U test and Chi-squared and Fisher Exact tests, as appropriate. 
Table I Demographics of 94 patients with juvenile idiopathic arthritis receiving adalimumab

\begin{tabular}{|c|c|c|c|}
\hline Variable & $\begin{array}{l}\text { JIA with uveitis } \\
(n=54)\end{array}$ & $\begin{array}{l}\text { JIA without uveitis } \\
(n=40)\end{array}$ & $P$ value \\
\hline Female, n (\%) & $37(69)$ & $26(65)$ & 0.72 \\
\hline Age at diagnosis, years, median (IQR) & $3(2,7)$ & $5(3,11)$ & 0.027 \\
\hline Age at end of study, years, median (IQR) & $15(14,18)$ & $16(11,18)$ & 0.67 \\
\hline Course type of arthritis, n (\%) & & & 0.003 \\
\hline Oligoarthritis & $14(26)$ & I (2) & \\
\hline Extended oligoarthritis & $17(3 \mid)$ & $12(30)$ & \\
\hline Polyarthritis: & $20(27)$ & $24(60)$ & \\
\hline Seronegative & 20 & 22 & \\
\hline Systemic JIA & $0(0)$ & $2(5)$ & \\
\hline Psoriatic arthritis & $2(4)$ & $0(0)$ & \\
\hline Spondyloarthropathy & I (2) & I (2) & \\
\hline HLA B27 present n (\%) ${ }^{\dagger}$ & $12(32)$ & $13(50)$ & 0.16 \\
\hline ANA present $\mathrm{n}(\%)^{\ddagger}$ & $36(7 I)$ & $9(31)$ & $<0.001$ \\
\hline
\end{tabular}

Notes: ${ }^{\dagger} \mid 5$ patients with uveitis and 2 without uveitis were missing data for HLA-B27; ${ }^{\ddagger}$ patient with uveitis and 3 without uveitis were missing data for the ANA studies. Abbreviations: ANA, antinuclear antibody; HLA, human leukocyte antigen; IQR, interquartile range; JIA, juvenile idiopathic arthritis.

\section{Results}

The main characteristics of the 94 patients receiving adalimumab at the beginning of the study are shown in Table 1 . The number of girls was slightly higher among the patients with uveitis (69\%) than those without it $(65 \%)$. The median age at onset of arthritis was lower in the patients with uveitis (median 3.0 years, interquartile range $2-7$ years) than that of patients without uveitis (median 5.0 years, interquartile range 3-11 years), but the difference was not statistically significant. Patients with uveitis were more often ANA-positive $(P<0.001)$.

The majority of patients with uveitis had oligoarthritis $(26 \%)$ or extended oligoarthritis (31\%). Among the patients without uveitis, the arthritis subtype was more aggressive; one patient had oligoarthritis, 12 had extended oligoarthritis, 22 had seronegative polyarthritis, two had seropositive polyarthritis, two had systemic onset arthritis, and one had spondyloarthropathy.

Adalimumab was stopped in 18 patients (five with associated uveitis) after a short period because of inefficacy or side effects, except for one patient who went to remission. At the end of the study, uveitis was under good clinical control in two thirds of patients, ie, $31 \%$ did not need any local treatment for uveitis and 35\% used only 1-2 corticosteroid

Table 2 Clinical control of uveitis in 54 patients treated with adalimumab

\begin{tabular}{ll}
\hline Effect of treatment on local uveitis & $\begin{array}{l}\text { Patients } \\
\mathbf{n}(\%)\end{array}$ \\
\hline No corticosteroid drops & $17(3 \mathrm{I})$ \\
I-2 corticosteroid drops/day & $19(35)$ \\
$\geq 3$ corticosteroid drops/day & $18(33)$ \\
\hline
\end{tabular}

drops per day. One third of the patients still had active uveitis and used at least three corticosteroid drops per day.

According to the SUN criteria, the response to adalimumab treatment was improved in $28 \%$ of patients, and worsened (two-step change in the amount of inflammation in the anterior chamber) in $13 \%$ of patients (Tables 2 and 3 ). Taking into account the 16 patients with uveitis whose response to adalimumab was moderate, an overall positive effect of adalimumab was seen in $57 \%$ of the patients with uveitis.

After 24 months of treatment with adalimumab, the proportion of patients with active arthritis decreased from $69 \%$ to $27 \%$ in the patients with uveitis, and from $93 \%$ to $59 \%$ in those without uveitis (Table 4). Systemic corticosteroid treatment could be stopped in $22 \%$ of patients with uveitis and in $11 \%$ of those without uveitis $(P=0.222$, data not shown).

Binocular visual acuity in the patients with juvenile idiopathic arthritis and uveitis was good in all but one patient who was legally blind because of severe early-onset chronic uveitis associated with juvenile psoriatic arthritis. Twenty-eight children (52\%) with juvenile idiopathic arthritis developed complications of uveitis, comprising five children who had incipient cataract, 21 who had undergone cataract surgery (intraocular lens implantation in 20 cases), five with

Table 3 Efficacy on uveitis according to Standardized Uveitis Nomenclature criteria in 54 patients*

\begin{tabular}{ll}
\hline Response to treatment & Patients, n (\%) \\
\hline Improved activity & $15(28)$ \\
Moderate response & $16(30)$ \\
No change & $16(30)$ \\
Worsening activity & $7(13)$ \\
\hline Note: *Adalimumab was stopped in 5 patients. &
\end{tabular}


Table 4 Effect of adalimumab on arthritis, and number of patients on adalimumab therapy at end of study

\begin{tabular}{llll}
\hline $\begin{array}{l}\text { Patients on adalimumab therapy at end of study } \\
\text { with signs of inflammation and swollen/active joints }\end{array}$ & $\begin{array}{l}\text { Patients with uveitis } \\
(\mathbf{n}=\mathbf{4 9 )}\end{array}$ & $\begin{array}{l}\text { Patients without uveitis } \\
(\mathbf{n}=\mathbf{2 7})\end{array}$ & \begin{tabular}{l}
$\boldsymbol{P}$ value \\
\hline At start of adalimumab, $\mathrm{n}(\%)$
\end{tabular} \\
At end of study & $34(69)$ & $25(93)$ & 0.023 \\
\hline
\end{tabular}

secondary glaucoma (Molteno implantation in three), and three with uveitis complicated by cystoid macular edema.

Most of the patients had been previously treated with more than one DMARD as monotherapy or as part of combination therapy (methotrexate, hydroxychloroquine, leflunomide, sulfasalazine, cyclosporin A, mycophenolate mofetil). At the end of the study, only four of 54 patients with juvenile idiopathic arthritis and uveitis remained on adalimumab monotherapy, and the rest were on combination therapy with prednisolone $(n=9)$, methotrexate $(n=34)$, leflunomide $(\mathrm{n}=9)$, cyclosporin A $(\mathrm{n}=7)$, azathioprine $(\mathrm{n}=1)$, sulfasalazine $(\mathrm{n}=4)$, or hydroxychloroquine $(n=2)$. The numbers of patients with uveitis on an antimetabolite and on systemic steroids at baseline and at the end of the study are shown in Table 5. Prednisolone therapy could be stopped in $22 \%$ of patients with uveitis. Of these patients, 29 had not responded to previous anti-TNF agents (etanercept or infliximab). Twenty-five patients were receiving adalimumab every fortnight at the end of the study, and 24 patients were receiving it weekly. The main clinical reason for weekly treatment was activity of uveitis.

\section{Discussion}

Adalimumab is a valuable option in the treatment of uveitis associated with refractory juvenile idiopathic arthritis. Our study was retrospective, but is one of the largest done in patients with treatment-resistant childhood uveitis treated with adalimumab.

Table 5 Number of patients with uveitis on an antimetabolite and systemic steroids at baseline and at end of study

\begin{tabular}{lll}
\hline & $\begin{array}{l}\text { Treatment at } \\
\text { baseline in patients } \\
\text { with uveitis } \\
(\mathbf{n = 5 4 )}\end{array}$ & $\begin{array}{l}\text { Treatment at } \\
\text { end of study } \\
\text { JIA with uveitis } \\
(\mathbf{n}=\mathbf{5 4})\end{array}$ \\
\hline Prednisolone & 20 & 9 \\
Methotrexate & 47 & 34 \\
Hydroxychloroquine & 5 & 2 \\
Cyclosporin A & 10 & 7 \\
Azathioprine & 2 & 1 \\
Leflunomide & 13 & 9 \\
Sulfasalazine & 8 & 4 \\
\hline
\end{tabular}

Abbreviation: JIA, juvenile idiopathic arthritis.
At the end of this study, disease activity improved in 15 of 54 patients with uveitis on adalimumab therapy ( $31 \%$ of those continuing with adalimumab), and disease activity worsened in seven patients (13\%) according to SUN criteria. However, improvement of uveitis was seen altogether in 31 out of 54 patients (57\%), because in another 16 patients with uveitis the response for uveitis was moderate, but did not reach the twofold change. In 16 cases, no change was seen according to SUN criteria. Clinical evaluation gave better results (improvement of uveitis in $66 \%$ of patients), because the use of SUN criteria in the evaluation of juvenile idiopathic arthritis-associated uveitis is difficult. The amount of anterior chamber cells is often quite small when the eye disease is still active. The two-fold difference is hard to achieve, because changes are quite small. In many cases, clinical evaluation by an experienced ophthalmologist may be better. The rate of favorable outcome was somewhat higher in previous studies with smaller numbers of patients, in which the activity of uveitis improved in 35\% (seven of 20 patients) according to a report by Tynjälä et al and in $89 \%$ according to a report by Biester et al. ${ }^{9,11}$

The type of arthritis was more severe in patients without uveitis. Any significant risk factors according to the type of arthritis were noted in this study. Both persistent and extended oligoarthritis groups were equally represented among the patients with juvenile idiopathic arthritis associated with uveitis. Interestingly, in a study from the UK consisting of 23 patients with juvenile idiopathic arthritis and uveitis, an association between patients with extended oligoarthritis and uveitis was noted, and patients with extended oligoarthritis also had a shorter interval from diagnosis of arthritis to development of uveitis. ${ }^{16}$ Five patients with juvenile idiopathic arthritis and uveitis had discontinued adalimumab therapy due to adverse events or inefficacy.

Our study was performed in clinical practice conditions, with a long period of follow-up by one ophthalmologist. Adalimumab was a good option in the treatment of patients with juvenile idiopathic arthritis and associated uveitis, often in combination with methotrexate and/or other DMARDs.

\section{Disclosure}

The authors report no conflicts of interest in this work. 


\section{References}

1. Kanski JJ. Juvenile arthritis and uveitis. Surv Ophthalmol. 1990;34: 253-267.

2. Kotaniemi K, Savolainen A, Karma A, Aho K. Major review. Recent advances in uveitis of juvenile idiopathic arthritis. Surv Ophthalmol. 2003;48:489-502.

3. Santos Lacomba M, Marcos Martín C, et al. Aqueous humor and serum tumor necrosis factor-alpha in clinical uveitis. Ophthalmic Res. 2001;33:251-255.

4. Smith JR, Levinson RD, Holland GN, et al. Differential efficacy of tumor necrosis factor inhibition in the management of inflammatory eye disease and associated rheumatic disease. Arthritis Rheum. 2001;45: 252-257.

5. Lovell D. Biological agents for the treatment of juvenile rheumatoid arthritis: current status. Paediatric Drugs. 2004;6:137-146.

6. Braun J, Baraliakos X, Listing J, Sieoer J. Decreased incidence of anterior uveitis in patients with ankylosing spondylitis treated with anti-tumor necrosis factor agents infliximab and etanercept. Arthritis Rheum. 2005;52:2447-2451.

7. Saurenmann RK, Levin AV, Rose JB, et al. Tumor necrosis factor $\alpha$ inhibitors in the treatment of childhood uveitis. Rheumatology. 2006;45:982-989.

8. Vazquez-Cobian L, Flynn T, Lehman T. Adalimumab therapy for childhood uveitis. J Pediatr. 2006;149:572-575.

9. Biester S, Deuter C, Michels H, et al. Adalimumab in the therapy of uveitis in childhood. Br J Ophthalmol. 2007;91:319-324.

10. Foeldvari I, Nielsen S, Kummerle-Deschner J, et al. Tumor necrosis factor-alpha blocker in treatment of juvenile idiopathic arthritisassociated uveitis refractory to second-line agents: results of a multinational survey. J Rheumatol. 2007;34:1146-1150.
11. Tynjälä P, Kotaniemi K, Lindahl P, et al. Adalimumab in juvenile idiopathic arthritis-associated chronic anterior uveitis. Rheumatology (Oxford). 2008;47:339-344.

12. Trachana M, Pratsidou-Gertsi P, Pardalos G, et al. Safety and efficacy of adalimumab treatment in Greek children with juvenile idiopathic arthritis. Scand J Rheumatol. 2011;40:101-107.

13. Petty RE, Southwood TR, Manners P, et al. International League of Associations for Rheumatology classification of juvenile idiopathic arthritis: second revision, Edmonton, 2001. J Rheumatol. 2004;31: 390-392.

14. Jabs DA, Nussenblatt RB, Rosenbaum JT; Standardization of Uveitis Nomenclature (SUN) Working Group. Standardization of uveitis nomenclature for reporting clinical data. Results of the first international workshop. Am J Ophthalmol. 2005;140:509-516.

15. Wallace CA, Ruperto N, Giannini E; Childhood Arthritis and Rheumatology Research Alliance; Pediatric Rheumatology International Trials Organization; Pediatric Rheumatology Collaborative Study Group. Preliminary criteria for clinical remission for select categories of juvenile idiopathic arthritis. J Rheumatology. 2004;31:2290-2294.

16. Sim KT, Venninig HE, Barrett S, et al. Extended oligoarthritis and other risk factors for developing JIA-associated uveitis under ILAR classification and its implication for current screening guideline. Ocul Immunol Inflamm. 2006;14:353-357.
Clinical Ophthalmology

\section{Publish your work in this journal}

Clinical Ophthalmology is an international, peer-reviewed journal covering all subspecialties within ophthalmology. Key topics include: Optometry; Visual science; Pharmacology and drug therapy in eye diseases; Basic Sciences; Primary and Secondary eye care; Patien Safety and Quality of Care Improvements. This journal is indexed on

Submit your manuscript here: http://www.dovepress.com/clinical-ophthalmology-journal

\section{Dovepress}

PubMed Central and CAS, and is the official journal of The Society of Clinical Ophthalmology (SCO). The manuscript management system is completely online and includes a very quick and fair peer-review system, which is all easy to use. Visit http://www.dovepress.com/ testimonials.php to read real quotes from published authors. 